\title{
Quantum walks based on an interferometric analogy
}

\author{
Mark Hillery $^{1}$, Janos Bergou ${ }^{1}$ and Edgar Feldman ${ }^{2}$ \\ ${ }^{1}$ Department of Physics, Hunter College of CUNY \\ 695 Park Avenue, New York, NY 10021 \\ ${ }^{2}$ Department of Mathematics \\ Graduate Center of the City University of New York \\ 365 Fifth Avenue, New York, NY 10016
}

November 2, 2018

\begin{abstract}
There are presently two models for quantum walks on graphs. The "coined" walk uses discrete time steps, and contains, besides the particle making the walk, a second quantum system, the coin, that determines the direction in which the particle will move. The continuous walks operate with continuous time. Here a third model for quatum walks is proposed, which is based on an analogy to optical interferometers. It is a discretetime model, and the unitary operator that advances the walk one step depnds only on the local structure of the graph on which the walk is taking place. This type of walk also allows us to introduce elements, such as phase shifters, that have no counterpart in classical random walks. Several examples are discussed.
\end{abstract}

\section{Introduction}

Random walks on graphs are the basis of a number of classical algorithms, Examples include, 2-SAT, graph connectivity, and finding satisfying assignments for Boolean formulas. As a result, it is natural to explore the quantum counterpart of a random walk, in the hope that it will be useful in the development of quantum algorithms. This has led to a number of studies. Quantum walks on the line were examined by Nayak and Vishwananth [1, and on the cycle by Aharonov, et al. [2]. The latter study also considered a number of properties of quantum walks on general graphs. Numerical simulations of walks in two and three dimensions were performed by Mackay, et al. 3]. Absorbing times and probabilities of quantum walks on the line were studied by several authors 4 [5. One of the main results to come from this work is that quantum walks spread 
faster than do classical ones. In particular, on the line, the standard deviation of the position of the particle making the walk increases linearly with the number of steps rather that with its square root as in the classical case. Walks on the hypercube have also been considered, and here the results are even more dramatic [6 7]. Kempe has shown that the hitting time for the walk from one corner of an $n$-bit hypercube to the opposite corner is polynomial in $n$ for a quantum walk, but exponential for a classical one. The quantum walk on the hypercube was subsequently used as the basis of a quantum search algorithm [8. The effect of decoherence on these walks has also been studied. Brun et al. showed how increasing dedoherence turns a quantum walk into a classical random walk 9]. Kendon and Treganna found that small amounts of decoherence can actually speed the convergence of the time-averaged probability distribution of a particle in a quantum walk to a uniform distribution [10].

The time steps in the quantum walks considered in these works are discrete. Continuous-time quantum walks have also been proposed [1]. It was shown that on a particular graph, the propagation between two properly chosen nodes is exponentially faster in the quantum case.

There is, at the moment, only one algorithm based on quantum walks on graphs, and it was proposed very recently by Childs, et al. 15. They constructed an oracle problem that can be solved by exponentially faster on a quantum computer than on a classical one by utilizing continuous quantum walks. The vertices of the graph are numbered (named), and there are two special vertices called the entrance and the exit. The problem is, given the name of the entrance, and the oracle, find the name of the exit. The oracle specifies the graph, which belongs to a particular set of possible graphs, by taking a binary number as its input, and either telling you that this number does not correspond to a vertex, or if it does, telling you the names of the adjacent vertices.

All of the discrete-time quantum walks are based on a particular model, the, "coined quantum walk", due to Watrous [16. In trying to formulate a quantum walk on a graph, the most natural thing to do is to let a set of orthonormal basis states correspond to the vertices of the graph. If a particle is in the state $|n\rangle$, that corresponds to its being located on vertex $n$. Trying to define a unitary evolution using this scheme soon leads to serious problems, as was first noted by Meyer [12. Watrous solved this problem by enlarging the Hilbert space in which the quantum walk takes place. How this scheme works is most easily seen by considering the quantum walk on a line. The vertices are labelled by integers, and, in addition, there is a quantum coin, which has two states, $|L\rangle$ and $|R\rangle$, corresponding to left and right, respectively. A basis for the Hilbert space describing this system is given by the states $|n\rangle \otimes \alpha\rangle$, where $n$ is an integer, and $\alpha$ is either $L$ or $R$. A step in this walk consists of applying the Hadamard operator, $H$, to the coin,

$$
\begin{aligned}
H|L\rangle & =\frac{1}{\sqrt{2}}(|L\rangle+|R\rangle) \\
H|R\rangle & =\frac{1}{\sqrt{2}}(|L\rangle-|R\rangle) .
\end{aligned}
$$


and then the operator

$$
V_{H}=S \otimes|R\rangle\left\langle R\left|+S^{\dagger} \otimes\right| L\right\rangle\langle L|,
$$

where $S$ is the shift operator, whose action is given by

$$
S|n\rangle=|n+1\rangle \quad S^{\dagger}|n\rangle=|n-1\rangle .
$$

The coined quantum walk can be extended in a simple way to regular graphs, i.e. those in which all vertices have the same number of edges emanating from them. When this is not true, things become more complicated, and it seems to be necessary to consdier the global structure of the graph in defining the walk. So far, no studies of discrete-time quantum walks on graphs that are not regular have appeared.

What we wish to propose here is a different type of discrete-time quantum walk. It is based on thinking about the graph as an interferometer. The vertices are optical elements known as $2 N$-ports, where $N$ is the number of edges meeting at the vertex, and the edges correspond to paths a photon can take through the interferometer. There is no quantum coin in these walks. The states are labelled by the edges rather than the vertices in the graph, and each edge has two states. If the edge is labelled $a b, a$ corresponding to one end and $b$ to the other, then one state is $a b$, corresponding to a photon going from $a$ to $b$, and the other is $b a$, corresponding to a photon going from $b$ to $a$. This approach is easily extended to arbitrary graphs; one simply writes down the transition rules for each vertex, and all of them taken together define a unitary operator that advances the walk one step. In addition, we can add elements to this walk that correspond to the addition of phase shifters to paths in an interferometer.

This model of a quantum walk on a graph is closely related to the optical networks considered by Törmä and Jex 13. They considered two-dimensional arrays of beam splitters and the propagation of photons through them. The horizontal motion of the photon in these networks corresponds to the time steps in a quantum walk, and the vertical position of the photon is just the position of the particle in the quantum walk. Note that these networks provide one with the opportunity to simulate the model of quantum walks proposed here with linear optics.

\section{Walk on the cycle}

Perhaps the simplest walk is that on a cycle or ring. Let us label the vertices by the numbers 0 through $N-1$, where the vertex $N$ is identical to 0 . That is, if we move one step forward from the vertex $N-1$, we end up at vertex 0 . The states of the system are $|j, k\rangle$, where $k=j \pm 1$, which can be thought of as a photon on the edge between vertices $j$ and $k$ going from $j$ to $k$. Because each edge has two states, and there are $N$ edges, the dimension of this space is $2 N$.

The vertices can be thought of as beam splitters. Consider what happens when a photon travelling in the horizontal direction hits a vertical beam splitter. The photon has a certain amplitude to continue in the direction it was going, i.e. to be transmitted, and an amplitude to be 
change its direction, i.e. to be reflected. The beam splitter has two input modes, the photon can enter from either the right or the left, and two output modes, the photon can leave heading either right or left. The beam splitter defines a unitary transformation between the input and output modes.

We now need to translate this analogy into transition rules for our quantum walk. Suppose we are in the state $|j-1, j\rangle$. If the photon is transmitted it will be in the state $|j, j+1\rangle$, and if reflected in the state $|j, j-1\rangle$. Let the transmission amplitude be $t$, and the reflection amplitude be $r$. We then have the transition rule

$$
|j-1, j\rangle \rightarrow t|j, j+1\rangle+r|j, j-1\rangle,
$$

where unitarity implies that $|t|^{2}+|r|^{2}=1$. The other possibility is that the photon is incident on vertex $j$ from the right, that is it is in the state $|j+1, j\rangle$. If it is transmitted it is in the state $|j, j-1\rangle$, and if it is reflected, it is in the state $|j, j+1\rangle$. Unitarity of the beam splitter transformation then gives us that

$$
|j+1, j\rangle \rightarrow t^{*}|j, j-1\rangle-r^{*}|j, j+1\rangle .
$$

These rules specify our walk.

The case $t=1$ and $r=0$ corresponds to free particle propagation; a "photon" in the state $|j, j+1\rangle$ simply moves one step to the right with each time step in the walk. If $r \neq 0$, then there is some amplitude to move both to the right and to the left. A physical system to which this is analogous is the motion of a particle in a periodic potential. The beam splitters can be thought of as scattering centers with the scattering resulting from a localized potential. As is well known, this leads to energy bands, and, as we shall soon see, a similar structure emerges in quantum walks on the cycle.

One way of approaching the study of the dynamics generated by this walk is to find the eigenvalues and eigenstates of the unitary transformation, $U$, that moves the system a single step. In order to do this, we first note that $U$ commutes with the translation operator, $T$, where

$$
T|j, j+1\rangle=|j+1, j+2\rangle \quad T|j+1, j\rangle=|j+2, j+1\rangle .
$$

This implies that these operators can be simultaneously diagonalized. The eigenvalues of $T$ are $e^{i \theta_{k}}$, where $\theta_{k}=2 \pi k / N$, and $k=0,1, \ldots N-1$. Each of these eigenvalues is doubly degenerate, and the two dimensional space of eigenvectors corresponding to $e^{i \theta_{k}}$ is spanned by

$$
\begin{aligned}
\left|u_{k+}\right\rangle & =\frac{1}{\sqrt{N}} \sum_{j=0}^{N-1} e^{i j \theta_{k}}|j, j+1\rangle \\
\left|u_{k-}\right\rangle & =\frac{1}{\sqrt{N}} \sum_{j=0}^{N-1} e^{i j \theta_{k}}|j+1, j\rangle .
\end{aligned}
$$

The eigenstates of $U$ are just linear combinations of $\left|u_{k+}\right\rangle$ and $\left|u_{k-}\right\rangle$. Defining

$$
\left|\psi_{k}\right\rangle=a_{k+}\left|u_{k+}\right\rangle+a_{k-}\left|u_{k-}\right\rangle,
$$


we find that the equation $U\left|\psi_{k}\right\rangle=\lambda\left|\psi_{k}\right\rangle$ becomes

$$
\left(\begin{array}{cc}
t e^{-i \theta_{k}} & -r^{*} \\
r & t^{*} e^{i \theta_{k}}
\end{array}\right)\left(\begin{array}{c}
a_{k+} \\
a_{k-}
\end{array}\right)=\lambda\left(\begin{array}{c}
a_{k+} \\
a_{k-}
\end{array}\right) .
$$

Expressing $t$ as $t=|t| e^{i \eta}$, we find that the eigenvalues are

$$
\lambda_{k \pm}=|t| \cos \left(\theta_{k}-\eta\right) \pm i\left[1-|t|^{2} \cos ^{2}\left(\theta_{k}-\eta\right)\right]^{1 / 2},
$$

and the corresponding eigenfunctions are given by

$$
\begin{aligned}
& a_{k+}^{(+)}=\frac{r^{*}}{\left[2 C_{k}\left(C_{k}+S_{k}\right)\right]^{1 / 2}} \\
& a_{k-}^{(+)}=\frac{-i\left(S_{k}+C_{k}\right)}{\left[2 C_{k}\left(C_{k}+S_{k}\right)\right]^{1 / 2}},
\end{aligned}
$$

for $\lambda_{k+}$, and

$$
\begin{aligned}
a_{k+}^{(-)} & =\frac{r^{*}}{\left[2 C_{k}\left(C_{k}-S_{k}\right)\right]^{1 / 2}} \\
a_{k-}^{(-)} & =\frac{i\left(C_{k}-S_{k}\right)}{\left[2 C_{k}\left(C_{k}-S_{k}\right)\right]^{1 / 2}},
\end{aligned}
$$

for $\lambda_{k-}$. Here we have defined

$$
\begin{aligned}
C_{k} & =\left[1-|t|^{2} \cos ^{2}\left(\theta_{k}-\eta\right)\right]^{1 / 2} \\
S_{k} & =|t| \sin \left(\theta_{k}-\eta\right) .
\end{aligned}
$$

One thing we notice immediately, is that for all of these eigenstates, the probability to be located on an edge, is the same for all edges, just $1 / N$. That means, that for any initial state, $\left|\Psi_{i n}\right\rangle$, the average probability distribution,

$$
p_{j}^{(m)}=\frac{1}{m} \sum_{k=0}^{m-1}\left(\left|\left\langle j, j+1\left|U^{k}\right| \Psi_{i n}\right\rangle\right|^{2}+\left|\left\langle j+1, j\left|U^{k}\right| \Psi_{i n}\right\rangle\right|^{2},\right.
$$

where $p_{j}^{(m)}$ is the average probability of being on the edge between $j$ and $j+1$ after $m$ steps, goes to a constant as $m \rightarrow \infty$, if all of the eigenvalues in Eq. (10) are distinct [2. This will be the case if $(N \eta) / \pi$ is not an integer.

\section{$3 \quad$ Walk on the line}

The quantum walk on the infinite line can be approached directly or as the limit of the walk on the cycle as $N$ goes to infinity. Because we have just found the eigenstates and eigenvalues for the walk on the cycle in the previous section, we shall adopt the latter course here. In particular, we want to examine what happens when we start the walk in the state $|0,1\rangle$. We shall present numerical results and then follow the approach developed by Nayak and Vishwanath to study the long time limit of the probability of being on the edge between the vertices $j$ and $j+1$. 


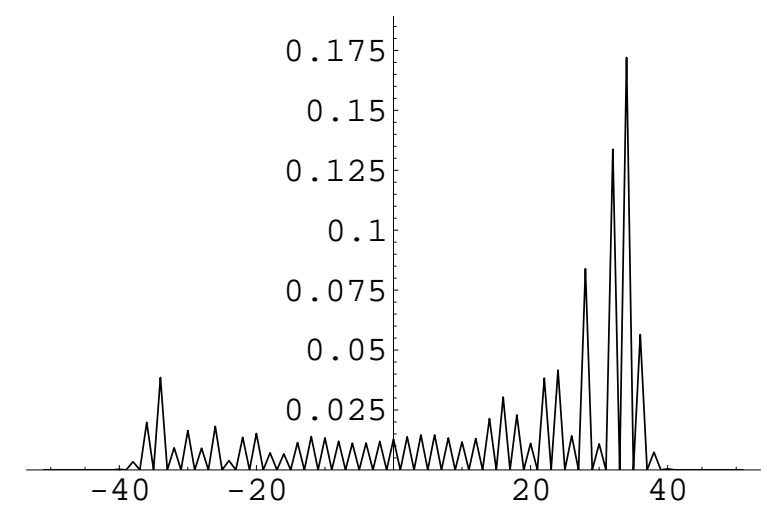

Figure 1: Probability distribution for quantum walk after 50 steps

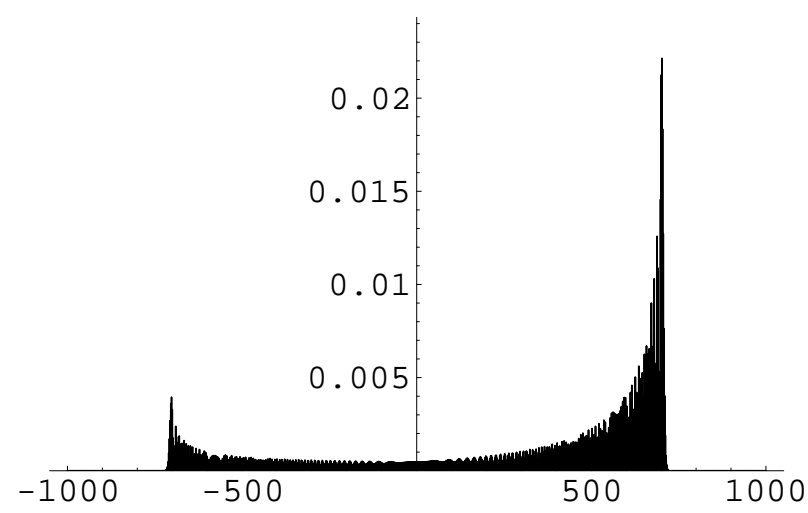

Figure 2: Probability distribution for quantum walk after 1000 steps

The probability distribution for the particle after $n$ steps can be computed in a straightforward manner. We display the results for the case $t=r=1 / \sqrt{2}$ and the initial state $|0,1\rangle$. In Figure 1 we have $n=50$ and in Figure 2, $n=1000$. Note that, as with the coined quantum walk, these probability distributions are not normal distributions. In addition, the region in which the probability of finding the particle is large is, roughly,in the case of $n=50$, between -35 and 35, and in the case $n=1000$, between -700 and 700 . In both cases this corresponds to the high probability region lying between $-|t| n$ and $|t| n$. This feature of the dynamics will be confirmed by our asymptotic analysis.

The asymptotic probabilities can be calculated as follows. Denoting the eigenstates corresponding to the eigenvalues $\lambda_{k+}$ and $\lambda_{k-}$ as $\left|\psi_{k+}\right\rangle$ and $\left|\psi_{k-}\right\rangle$, respectively, we find that the wave function of the particle 
executing the walk, $|\Psi(\tau)\rangle$ is, after $\tau$ steps,

$$
\begin{aligned}
|\Psi(\tau)\rangle & =\sum_{k=0}^{N-1} \sum_{s= \pm} \lambda_{k, s}^{\tau}\left|\psi_{k, s}\right\rangle\left\langle\psi_{k, s} \mid 0,1\right\rangle \\
& =\frac{1}{\sqrt{N}} \sum_{k=0}^{N-1}\left(a_{k+}^{(+) *} \lambda_{k+}^{\tau}\left|\psi_{k+}\right\rangle+a_{k+}^{(-) *} \lambda_{k-}^{\text {tau }}\left|\psi_{k-}\right\rangle\right)
\end{aligned}
$$

where, as mentioned in the previous paragraph, the initial state is taken to be $|0,1\rangle$. The amplitudes to be in the states $|j . j+1\rangle$ and $|j+1, j\rangle$ at time $\tau$ are given by

$$
\begin{aligned}
\langle j, j+1 \mid \Psi(\tau)\rangle & =\frac{1}{N} \sum_{k=0}^{N-1} e^{i j \theta_{k}}\left(\lambda_{k+}^{\tau}\left|a_{k+}^{(+)}\right|^{2}+\lambda_{k-}^{\tau}\left|a_{k+}^{(-)}\right|^{2}\right) \\
\langle j+1, j \mid \Psi(\tau)\rangle & =\frac{1}{N} \sum_{k=0}^{N-1} e^{i j \theta_{k}}\left(\lambda_{k+}^{\tau} a_{k+}^{(+) *} a_{k-}^{(+)}+\lambda_{k-}^{\tau} a_{k+}^{(-) *} a_{k-}^{(-)}\right) .
\end{aligned}
$$

The probability of being on the edge between vertices $j$ and $j+1$ at time $\tau, p(j, j+1 ; \tau)$, is

$$
p(j, j+1 ; \tau)=|\langle j, j+1 \mid \Psi(\tau)\rangle|^{2}+|\langle j+1, j \mid \Psi(\tau)\rangle|^{2} .
$$

In order to go the the $N \rightarrow \infty$ limit, we need to define a number of functions of the continuous variable $\theta$ rather than expressing them as functions of the discrete variable $\theta_{k}$. We first define

$$
\begin{array}{r}
C(\theta)=\left[1-|t|^{2} \cos ^{2}(\theta-\eta)\right]^{1 / 2} \\
S(\theta)=|t| \sin (\theta-\eta) .
\end{array}
$$

The eigenvalues also become functions of $\theta$, and we shall express them as

$$
\lambda_{ \pm}(\theta)=e^{i \omega_{ \pm}(\theta)},
$$

where $0 \leq \omega_{+}(\theta) \leq \pi$ and

$$
\omega_{+}(\theta)=\tan ^{-1}\left(\frac{|t| \cos (\theta-\eta)}{C(\theta)}\right),
$$

and $-\pi \leq \omega_{-}(\theta) \leq 0$ and

$$
\omega_{-}(\theta)=\tan ^{-1}\left(-\frac{|t| \cos (\theta-\eta)}{C(\theta)}\right) .
$$

We can now proceed to take the $N \rightarrow \infty$ limit of the sums appearing in Eqs. (16). For the sums appearing in the first of these equations we have

$$
\begin{aligned}
\frac{1}{N} \sum_{k=0}^{N-1} e^{i j \theta_{k}} \lambda_{k+}^{\tau}\left|a_{k+}^{(+)}\right|^{2} \rightarrow & \int_{0}^{2 \pi} d \theta e^{i\left[j \theta+\tau \omega_{+}(\theta)\right]} \frac{|r|^{2}}{4 \pi C(\theta)[C(\theta)+S(\theta)]} \\
\frac{1}{N} \sum_{k=0}^{N-1} e^{i j \theta_{k}} \lambda_{k-}^{\tau}\left|a_{k+}^{(-)}\right|^{2} \rightarrow & \int_{0}^{2 \pi} d \theta e^{i\left[j \theta+\tau \omega_{-}(\theta)\right]} \\
& \frac{|r|^{2}}{4 \pi C(\theta)[C(\theta)-S(\theta)]}
\end{aligned}
$$


The sums in the second equation become

$$
\begin{array}{ll}
\frac{1}{N} \sum_{k=0}^{N-1} e^{i j \theta_{k}} \lambda_{k+}^{\tau} a_{k+}^{(+) *} a_{k-}^{(+)} & \rightarrow-\int_{0}^{2 \pi} d \theta e^{i\left[j \theta+\tau \omega_{+}(\theta)\right]} \frac{i r}{4 \pi C(\theta)} \\
\frac{1}{N} \sum_{k=0}^{N-1} e^{i j \theta_{k}} \lambda_{k-}^{\tau} a_{k+}^{(-) *} a_{k-}^{(-)} & \rightarrow \int_{0}^{2 \pi} d \theta e^{i\left[j \theta+\tau \omega_{-}(\theta)\right]} \frac{i r}{4 \pi C(\theta)} .
\end{array}
$$

We are now going to analyze these integrals in the large $\tau$ limit by using the method of stationary phase. This will be done in two different ways. In the first, $j$ will be fixed and $\tau$ will go to infinity. In the second, we shall set $j=\alpha \tau$, and then let $\tau$ go to infinity. Some of the details of this analysis are given in the appendix. Here we shall just present the results. In the case of fixed $j$ we have that

$$
\begin{aligned}
p(j, j+1 ; \tau) \sim & \frac{|r|}{\pi \tau|t|}\left\{\left[1+(-1)^{j+\tau}\right] \cos ^{2}(\tau \mu+\pi / 4)\right. \\
& \left.+\left[1-(-1)^{j+\tau}\right] \sin ^{2}(\tau \mu+\pi / 4)\right\}
\end{aligned}
$$

where $0 \leq \mu \leq \pi / 2$, and

$$
\mu=\tan ^{-1}\left(\frac{|r|}{|t|}\right) .
$$

We note that this implies that for any interval located symmetrically about the origin, the probability of being in that interval goes like $1 / \tau$, whereas for a classical random walk starting at the origin, it would go like $1 / \sqrt{\tau}$. This implies that, as with the coined quantum walk, this quantum walk spreads faster than a classical one. In the case that $j=\alpha \tau$, we find that there are stationary phase points only if $\alpha \leq|t|$. That means that for $\alpha>|t|, p(j, j+1 ; \tau)=p(\alpha \tau, \alpha \tau+1 ; \tau)$ decreases faster than any inverse power of $\tau$. For $\alpha<|t|$ we have that $p(j, j+1 ; \tau)$ goes like $1 / \tau$. Therefore, it is most probable that the particle is located in the region $|j| \leq|t| \tau$, and we can say that the allowed region for the particle expands with speed $|t|$.

\section{Relation between quantum walks}

We now have two different quantum walks on the line, the coined walk, where one moves between vertices, and, what we shall call the edge walk, where the quantum particle making the walk resides on the edges between the vertices. It would be useful to know if the two different walks are related. In this section we shall show that they are unitarily equivalent. It should be emphasized that this result will only be demonstrated for the line, whether it holds for more general graphs is not known. Presently, no description of a coined walk for a general graph has appeared.

Let us begin by examining the Hilbert spaces for the two different quantum walks. The canonical orthonormal basis states of the Hilbert space for the coined walk on the line are given by $\{|j\rangle \otimes|R\rangle,|j\rangle \otimes|L\rangle \mid j \in Z\}$, where the state $|j\rangle$ corresponds to the $j$ th vertex, and $|r\rangle$ and $|L\rangle$ are the coin states. The Hilbert space in which this walk takes place is just 
$L^{2}(Z) \otimes L^{2}\left(Z_{2}\right)$. The canonical orthonormal basis of the Hilbert space for the edge walk is $\{|j, j+1\rangle,|j+1, j\rangle \mid j \in Z\}$, and the Hilbert space itself is $L^{2}\left(Z \times Z_{2}\right)$, which is identical to $L^{2}(Z) \otimes L^{2}\left(Z_{2}\right)$.

Let us now move to the dynamics. The unitary operator, $V$, that advances the coined walk one step is given by

$$
V=\left(S \otimes|R\rangle\left\langle R\left|+S^{\dagger} \otimes\right| L\right\rangle\langle L|\right)(I \otimes G),
$$

where $G \in U(2)$ is a generalized "coin-flip" operator, and is given by

$$
\begin{aligned}
G|R\rangle & =t|R\rangle+r|L\rangle \\
G|L\rangle & =-r^{*}|R\rangle+t^{*}|L\rangle .
\end{aligned}
$$

The unitary operator, $U$, that advances the edge walk one step was given in Section 2, and is

$$
\begin{aligned}
& U|j-1, j\rangle=t|j, j+1\rangle+r|j, j-1\rangle \\
& U|j+1, j\rangle=t^{*}|j, j-1\rangle-r^{*}|j, j+1\rangle .
\end{aligned}
$$

Define the unitary operator $\hat{E}$, which takes $L^{2}\left(Z \times Z_{2}\right)$ into itself, and is given explicitly by

$$
\begin{aligned}
\hat{E}|j-1, j\rangle & =|j\rangle \otimes|R\rangle \\
\hat{E}|j+1, j\rangle & =|j\rangle \otimes|L\rangle .
\end{aligned}
$$

We find that

$$
V \hat{E}=\hat{E} U,
$$

so that at the level of amplitudes, the two walks are unitarily equivalent.

There is, however, a difference in the probabilities. In the coined walk, the probability to be on vertex $j$ is given by combining (taking the squares of the magnitudes and adding) the amplitudes for the states $|j\rangle \otimes|R\rangle$ and $|j\rangle \otimes|L\rangle$. Under the mapping $\hat{E}^{-1}$, these states correspond to states on different edges, $|j-1, j\rangle$ and $|j+1, j\rangle$, respectively. However, the probabilities in the edge walk are computed by combining the amplitudes for being on the same edge, e.g. those for $|j-1, j\rangle$ and $|j, j-1\rangle$. Therefore, there will be a difference in the probabilites for the two walks. This can be seen explcitly if we examine the probability distribution for the case $t=r=1 / \sqrt{2}$. We again start in the state $|1,0\rangle$, let the walk go for 50 steps, but now compute the probability that the particle is on a vertex, instead of computing the probability that it is on an edge. The result is shown in Figure 3. By comparing this figure to Fig. 1, we see that the overall shape of the probability distributions is similar, but the details are different.

\section{Phase shifters}

Going back to the interferometer analogy, we note that we can add a new element to quantum walks that has no analogue in classical random walks. Interferometers are made up of multiports and phase shifters; a phase shifter imparts a constant phase to a photon that passes through 


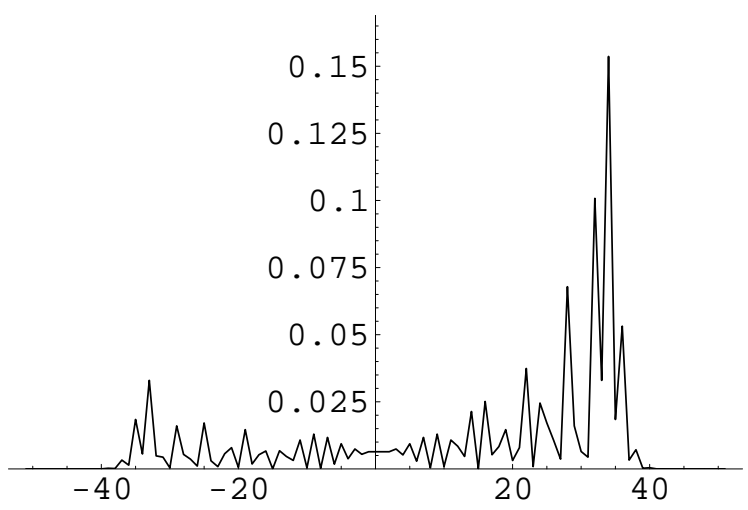

Figure 3: Probability distribution for $n=50$, and particle on vertices instead of edges.

it. Suppose we were to put a phase shifter that imparts a phase shift of $\phi$ just before the $j^{\text {th }}$ vertex. The transition rules for the states adjacent to this vertex are modified, while the rules for all other states are unaffected. In particular, we now have

$$
\begin{aligned}
& |j-1, j\rangle \rightarrow t e^{i \phi}|j, j+1\rangle+r e^{2 i \phi}|j, j-1\rangle \\
& |j+1, j\rangle \rightarrow-r^{*}|j, j+1\rangle+t^{*} e^{i \phi}|j, j-1\rangle .
\end{aligned}
$$

Insertion of a phase shifter into an edge can change the properties of a quantum walk, because it changes how different paths interfere.

One system that allows us to see their effect on the average probability distribution is a modified walk on a cycle. Suppose that the number of vertices is even, and that we put a phase shifter in all of the edges whose left end is an even numbered vertex, i.e. every second edge has a phase shifter in it. This system is exactly solvable, and by examining its eigenstates, we shall see how the average probability distributions it gives rise to depend on the value of $\phi$.

The unitary operator that advances this walk one step acts in the following way if $j$ is even

$$
\begin{aligned}
U_{2}|j, j+1\rangle & =t e^{i \phi}|j+1, j+2\rangle+r e^{2 i \phi}|j+1, j\rangle \\
U_{2}|j+1, j\rangle & =t^{*}|j, j-1\rangle-r^{*}|j, j+1\rangle,
\end{aligned}
$$

and if $j$ is odd, then

$$
\begin{aligned}
U_{2}|j, j+1\rangle & =t|j+1, j+2\rangle+r|j+1, j\rangle \\
U_{2}|j+1, j\rangle & =t^{*} e^{i \phi}|j, j-1\rangle-r^{*}|j, j+1\rangle .
\end{aligned}
$$

This operator commutes with translations by two steps, i.e. with the operator $T^{2}$. The eigenstates of $T^{2}$ are given by

$$
\left|u_{k+}^{(e)}\right\rangle=\sqrt{\frac{2}{N}} \sum_{j=0, \text { even }}^{N-1} e^{i j \theta_{k}}|j, j+1\rangle
$$




$$
\begin{aligned}
\left|u_{k+}^{(o)}\right\rangle & =\sqrt{\frac{2}{N}} \sum_{j=0, \text { odd }}^{N-1} e^{i j \theta_{k}}|j, j+1\rangle \\
\left|u_{k-}^{(e)}\right\rangle & =\sqrt{\frac{2}{N}} \sum_{j=0, \text { even }}^{N-1} e^{i j \theta_{k}}|j+1, j\rangle \\
\left|u_{k-}^{(o)}\right\rangle & =\sqrt{\frac{2}{N}} \sum_{j=0, \text { odd }}^{N-1} e^{i j \theta_{k}}|j+1, j\rangle .
\end{aligned}
$$

Each of these states has the eigenvalue $\exp \left(-2 i \theta_{k}\right)$. Eigenstates of $U_{2}$ are just linear combinations of the above states. In particular, expressing the eigenstate of $U_{2},\left|\psi_{k}^{(2)}\right\rangle$, as

$$
\left|\psi_{k}^{(2)}\right\rangle=a_{k+}\left|u_{k+}^{(e)}\right\rangle+a_{k-}\left|u_{k-}^{(e)}\right\rangle+b_{k+}\left|u_{k+}^{(o)}\right\rangle+b_{k-}\left|u_{k-}^{(o)}\right\rangle,
$$

the eigenvalue equation $U_{2}\left|\psi_{k}^{(2)}\right\rangle=\lambda\left|\psi_{k}^{(2)}\right\rangle$ becomes

$$
\left(\begin{array}{cccc}
0 & 0 & -r^{*} & t e^{-i \theta_{k}} \\
0 & 0 & t^{*} e^{i \theta_{k}} & r \\
r e^{2 i \phi} & t^{*} e^{i\left(\theta_{k}+\phi\right)} & 0 & 0 \\
t e^{i\left(\phi-\theta_{k}\right)} & -r^{*} & 0 & 0
\end{array}\right)\left(\begin{array}{c}
a_{k+} \\
b_{k-} \\
a_{k-} \\
b_{k+}
\end{array}\right)=\lambda\left(\begin{array}{c}
a_{k+} \\
b_{k-} \\
a_{k-} \\
b_{k+}
\end{array}\right) .
$$

The eigenvalues satisfy the equation

$$
\lambda^{4}+\lambda^{2}\left[|r|^{2}\left(1+e^{2 i \phi}\right)-e^{i \phi}\left(t^{* 2} e^{2 i \theta_{k}}+t^{2} e^{-2 i \theta_{k}}\right)\right]+e^{2 i \phi}=0 .
$$

The eigenstates of this system no longer give rise to constant probability distributions; the probabilities of being on an even edge (an edge whose left-most vertex is even) and an odd edge (an edge whose left-most vertex is odd) are, in general, different. If $\phi=0$, these probabilities are the same, but if $\phi=\pi / 2$, then this is no longer the case. In the latter case we find that

$$
\lambda^{2}=i|t|^{2} \cos \left(2 \theta_{k}-2 \eta\right) \pm\left[1-|t|^{4} \cos ^{2}\left(2 \theta_{k}-2 \eta\right)\right]^{1 / 2} .
$$

Choosing the plus sign in the above equation, we find that for an eigenfunction corresponding to $\theta_{k}-\eta=\pi / 4$, we have for the ratio of the probability of being on an even edge to being on an odd one

$$
\frac{p_{\text {even }}}{p_{\text {odd }}}=\frac{1+|r|^{2}}{1-|r|^{2}} .
$$

If $\theta_{k}-\eta$ cannot be exactly equal to $\pi / 4$ because of the values of $N$ or $\eta$, then for $\theta_{k}-\eta$ close to $\pi / 4$ the ratio of even-edge to odd-edge probabilities will be approximately given by the above equation. This ratio is not generally equal to one, which means that the average probability distribution to which a given initial state converges will not be constant. The introduction of the phase shifters has changed the character of the quantum walk.

These changes can also be seen by calculating the probability distributions after a finite number of steps. This is done for 50 steps and for 


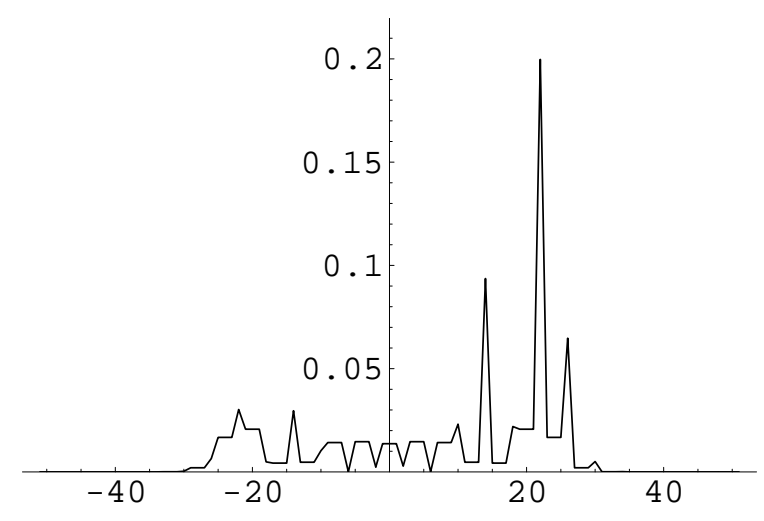

Figure 4: Probability distribution for $n=50$ and $\phi=\pi / 2$.

the case $t=r=1 / \sqrt{2}$ and $\phi=\pi / 2, \pi / 3$ in the following figures. The initial state is, as before, $|0,1\rangle$. These can be compared to Fig. 1, which corresponds to the case $\phi=0$. It can be seen that the introduction of the phase shifter greatly changes the character of the probability distribution. Note that particularly for the case of $\phi=\pi / 2$, the size of the region in which it is very likely that the particle will be found is smaller than when $\phi=0$. For a small number of steps, it is easy to verify by hand that destructive interference in the $\phi=\pi / 2$ case makes the walk spread more slowly than when $\phi=0$, and the numerical results indicate that this feature persists for at least 50 steps.

If we extend this walk to the infinite line, the difference caused by the phase shifters can be seen in the asymptotic behavior. For both $\phi=0$ and $\phi=\pi / 2$ the size of the region in which it is most likely to find the particle grows linearly with the number of steps, but the "speed" is different. We saw that in the case $\phi=0$ the probability distribution $p(j, j+1: \tau)$ falls off rapidly for $|j|>|t| \tau$. If $\phi=\pi / 2$, it falls off rapidly for $|j|>|t|^{2} \tau$, which means that the size of the high probability region is smaller in this case. We see yet again, that phase effects, which do not exist in classical random walks, can significantly influence the behavior of quantum walks.

\section{Probability current}

In standard quantum mechanics, it is possible to define a probability current density. In one dimension, if the wave function of the particle is $\psi(x, t)$, then the probability current density is given by

$$
j(x, t)=\frac{1}{2 i m}\left[\psi^{*}(x, t) \frac{d}{d x} \psi(x, t)-\psi(x, t) \frac{d}{d x} \psi^{*}(x, t)\right],
$$




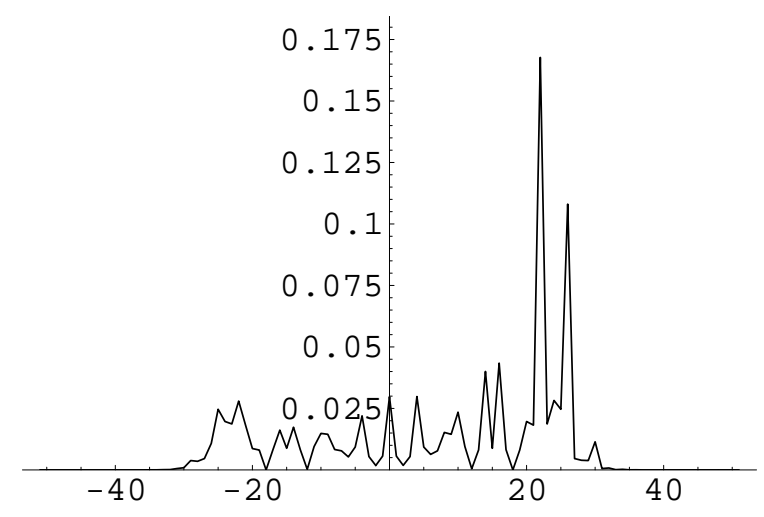

Figure 5: Probability distribution for $n=50$ and $\phi=\pi / 3$.

where $m$ is the mass of the particle, and we are using units in which $\hbar=1$. This current has the property that

$$
\frac{\partial}{\partial t} \int_{x_{1}}^{x_{2}}|\psi(x, t)|^{2}=-\left[j\left(x_{2}, t\right)-j\left(x_{1}, t\right)\right],
$$

that is, the change in the probability of the particle being in a particular region is given by the net flow of probability into the region. For an eigenstate of the Hamiltonian, the probability density $|\psi(x, t)|^{2}$ is independent of time, so that the probability current density is a constant. We would like to show that there is a quantity similar to the probability current density for quantum walks.

Suppose that the state of the walk on the cycle is given by

$$
|\Psi\rangle=\sum_{j=0}^{N-1}\left(c_{j, j+1}|j, j+1\rangle+c_{j+1, j}|j+1, j\rangle\right) .
$$

Define the probability current at the $k^{\text {th }}$ vertex to be

$$
J_{k}=\left(\begin{array}{ll}
c_{k+1, k}^{*} & c_{k-1, k}^{*}
\end{array}\right)\left(\begin{array}{cc}
|t|^{2} & t r \\
t^{*} r^{*} & -|t|^{2}
\end{array}\right)\left(\begin{array}{c}
c_{k+1, k} \\
c_{k-1, k}
\end{array}\right) .
$$

We find that, if $\Delta P_{k, k+1}$ is the change in the probability of being on the edge between vertices $k$ and $k+1$ in one step of the walk, then

$$
\Delta P_{k, k+1}=J_{k+1}-J_{k},
$$

which is the discrete analogue of Eq. (41). For an eigenstate of the walk, this current, $J_{k}$, must be independent of $k$. The above equation holds even if the transmission and reflection amplitudes are different for each beam splitter, i.e. they depend on $k$, and also if phase shifters are present. In that case, if $t_{k}$ is the transmission amplitude at vertex $k, r_{k}$ the reflection 
amplitude, and $\phi_{k}$ the phase shift of the phase shifter just to the left of vertex $k$, the current at this vertex is give by

$$
J_{k}=\left(c_{k+1, k}^{*} c_{k-1, k}^{*}\right)\left(\begin{array}{cc}
\left|t_{k}\right|^{2} & t r e^{i \phi_{k}} \\
t^{*} r^{*} e^{-i \phi_{k}} & -\left|t_{k}\right|^{2}
\end{array}\right)\left(\begin{array}{c}
c_{k+1, k} \\
c_{k-1, k}
\end{array}\right) .
$$

We can use this fact to demonstrate a general property of eigenstates of certain kinds of walks on a line. Suppose that all of the beam splitters located at vertices $k<0$ and $k>N$ have transmission amplitude $t=1$ and reflection amplitude $r=0$. The beam splitters for $0 \leq k \leq N$ can have any value of transmission amplitude and reflection amplitude, and these values can vary from vertex to vertex. We shall refer to the vertices between 0 and $N$ as the scattering region. The problem we are considering is analogous to the scattering of a particle moving in one dimension off of a potential, which is nonzero only in a bounded interval. From the point of view of a walk, we may be interested in a walk that starts to the left of the scattering region in a right-moving state, and finding out how long it takes to get through the scattering region.

The eigenstates of this type of walk are of two types. The first of a particle coming in from the left, its reflected amplitude, and a transmitted amplitude to the right of the scattering region. The second consists of a particle coming in from the right, its reflected amplitude, and a transmitted amplitude to the left of the scattering region. We shall consider the first type, which can be expressed as

$$
|\Psi\rangle=\sum_{j=-\infty}^{N-1}\left(c_{j, j+1}|j, j+1\rangle+c_{j+1, j}|j+1, j\rangle\right)+\sum_{j=N}^{\infty} c_{j, j+1}|j, j+1\rangle .
$$

Setting the eigenvalue, $\lambda$, equal to $\exp (-i \theta)$, the equation $U|\Psi\rangle=\lambda|\Psi\rangle$ gives us that

$$
\begin{array}{cc}
c_{j, j+1}=e^{i(j+1) \theta} c_{-1,0} & \text { for } j \leq-1 \\
c_{j+1, j}=e^{-i(j+1) \theta} c_{0,-1} & \text { for } j \leq-1 \\
c_{j, j+1}=e^{i(j-N) \theta} c_{N, N+1} & \text { for } j \geq N .
\end{array}
$$

The amplitude $c_{-1,0}$ can be thought of as the amplitude of the incoming wave, $c_{0,-1}$ the amplitude of the reflected wave, and $c_{N, N+1}$ the amplitude of the transmitted wave. We can find a condition that these quantities must satisfy, if we make use of the fact that $J_{-1}=J_{N+1}$. This gives us that

$$
\left|c_{0,-1}\right|^{2}+\left|c_{N, N+1}\right|^{2}=\left|c_{-1,0}\right|^{2}
$$

where we have used the fact that $\left|c_{-2,-1}\right|^{2}=\left|c_{-1,0}\right|^{2}$. Defining the reflection coefficient of the scattering region to be $R=\left|c_{0,-1}\right|^{2} /\left|c_{-1,0}\right|^{2}$ and the transmission coefficient to be $T=\left|c_{N, N+1}\right|^{2} /\left|c_{-1,0}\right|^{2}$, then the above equation can be expressed as $R+T=1$.

\section{Vertices with more than two edges}

So far we have only considered vertices at which two edges meet, but if we are to construct graphs more complicated than lines, we need to see 
how a vertex with more that two edges emanating from it behaves. We shall look at two examples, one with three edges, and another with an arbitrary number.

A vertex with three edges emanating from it, which is inspired by the optical multiport known as the tritter [14, can be described as follows. Let us label the vertex with three edges meeting at it by $O$, and the opposite ends of the edges by $A, B$, and $C$. The ingoing states for this vertex are $|A O\rangle,|B O\rangle$, and $|C O\rangle$, and the outgoing states are $|O A\rangle,|O B\rangle$, and $|O C\rangle$. Setting $z=\exp (2 \pi i / 3)$, we have for the transition rules

$$
\begin{aligned}
& |A O\rangle \rightarrow \frac{1}{\sqrt{3}}(|O A\rangle+|O B\rangle+|O C\rangle) \\
& |B O\rangle \rightarrow \frac{1}{\sqrt{3}}\left(z^{*}|O A\rangle+|O B\rangle+z|O C\rangle\right) \\
& |C O\rangle \rightarrow \frac{1}{\sqrt{3}}\left(z^{*}|O A\rangle+z|O B\rangle+|O C\rangle\right) .
\end{aligned}
$$

This vertex has the property that an incoming particle is equally likely to exit through each edge. However, note that because the incoming states from different edges behave differently with regard to their phases, the use of this vertex requires the labelling of edges. In this particular case, only one of the edges needs to be labelled. If we attach a label to $A O$, we interpret it to mean that if the input state is along either of the other two edges, then the output with a phase factor of $z^{*}$ is along the labelled edge. For any edge, if the input state is along this edge, the part of the output state along the same edge has a phase factor of one.

It is possible to define vertices that do not require the labelling of edges, though the cost is that the probabilities of exiting through each of the edges are no longer the same. This particular kind of vertex is very closely related to the quantum coin used in the walk on the hypercube 6] 7. Let the vertex at which all of the edges meet be labelled by $O$, and the opposite ends of the edges be labelled by the numbers 1 through $n$. For any input state, $|k O\rangle$, where $k$ is an integer between 1 and $n$, the transition rule is that the amplitude to go the output state $|O k\rangle$ is $r$, and the amplitude to go to any other output state is $t$. That is, the amplitude to be reflected is $r$, and the amplitude to be transmitted through any of the other edges is $t$. Unitarity places two conditions on these amplitudes

$$
\begin{aligned}
(n-1)|t|^{2}+|r|^{2} & =1 \\
(n-2)|t|^{2}+r^{*} t+t^{*} r & =0 .
\end{aligned}
$$

As an example, for the case $n=3$, possible values of $r$ and $t$ are $r=-1 / 3$ and $t=2 / 3$. Because each of the edges in this vertex behaves in the same way, they are equivalent to each other and no labelling is necessary.

In order to construct a walk for a general graph, one chooses a unitary operator for each vertex, i.e. one that maps the states coming into a vertex to states leaving the same vertex. One step of the walk consists of the combined effect of all of these operations; the overall unitary operator, $U$, that advances the walk one step is constructed from the local operators for each vertex. Explicitly, the edge state $|a b\rangle$, which can be interpreted as going from vertex $a$ to vertex $b$, will go to the state $U_{b}|a b\rangle$ after one 
step, where $U_{b}$ is the operator corresponding to vertex $b$. This prescription guarantees that the overall operation is unitary, in particular, $U$ acting on any other edge state $|c d\rangle$ will give a state orthogonal to $U|a b\rangle$. If $d=b$, then $|a b\rangle$ and $|c d\rangle$ will be mapped onto the same set of states (the states leaving vertex $b$ ), but the unitarity of $U_{b}$ will ensure that $U|a b\rangle$ and $U|c d\rangle$ are orthogonal. If $d \neq b$, then $U$ maps $|a b\rangle$ and $|c d\rangle$ onto different sets of states, and the so results are then orthogonal. Therefore, as the edge states make up an orthonormal basis of the Hilbert space in which the walk occurs, and $U$ maps this basis to another orthonormal basis, it is unitary.

\section{Conclusion}

Quantum walks on graphs seem promising for the development of algorithms, because they spread over a graph faster than does a classical walk, and can thereby explore the structure of the graph faster than can a classical random walk. Here we have discussed a discrete quantum walk that is based on an analogy to optical interferomenters. The vertices act as optical multiports and phase shifters can be inserted into the edges. As we have seen, the behavior of this type of walk depends on both types of elements. The advantage of this type of quantum walk is that it can easily be defined for any graph.

In this paper, we have, for the most part, confined our attention to the quantum walk on the line. We found that the probability distribution of the particle making the walk spreads linearly with the number of steps, and with a "speed" given by $|t|$. In addition, there is a probability current, whose "divergence" gives the probability flowing into an edge. This allowed us to define reflection and transmission coefficients for one-dimensional graphs.

The extension of these results to more general graphs is clearly the next step. It has been shown how definine a quantum walk for any graph, but the properties of these walks have yet to be explored.

\section{Acknowledgments}

This research was supported in part by the National Science Foundation under grant number PHY-0139692 and by the Office of Naval Research under grant number N00014-94J-1233. We would also like to thank Jozef Škvarček for preliminary numerical work on this problem.

\section{Appendix}

Here we want to explicitly show how the asymptotic analysis on the integrals in section III. We shall show how to do the analysis for the first integral in Eq. (22), and the procedure for the others is similar.

We shall first consider the case when $j$ is fixed. Then the stationary phase points are the solutions of the equation $\omega_{+}^{\prime}(\theta)=0$, and we find the two solutions $\theta=\eta$ and $\theta=\eta+\pi$. We also find that $\omega_{+}^{\prime \prime}(\eta)=|t| /|r|$ and 
$\omega_{+}^{\prime \prime}(\eta+\pi)=-|t| /|r|$. In addition, $\omega_{+}(\eta)=\mu$ and $\omega_{+}(\eta+\pi)=\pi-\mu$. Inserting these values into the standard formula for stationary phase [17, we find that

$$
\begin{aligned}
\int_{0}^{2 \pi} d \theta e^{i\left[j \theta+\tau \omega_{+}(\theta)\right]} \frac{|r|^{2}}{4 \pi C(\theta)[C(\theta)+S(\theta)]} & \sim \frac{1}{2}\left(\frac{|r|}{2 \pi \tau|t|}\right)^{1 / 2} e^{i j \eta} \\
\left(e^{i(\tau \mu+\pi / 4)}+(-1)^{j+\tau} e^{-i(\tau \mu+\pi / 4)}\right) . &
\end{aligned}
$$

Let us now consider the case when $j=\alpha \tau$. The stationary phase points are now given by the solutions of

$$
\omega_{+}^{\prime}(\theta)=\frac{|t| \sin (\theta-\eta)}{\left[1-|t|^{2} \cos ^{2}(\theta-\eta)\right]^{1 / 2}}=-\alpha .
$$

For this equation to have any solutions, we find that $\alpha \leq|t|$. If this condition is not satisfied, there are no stationary phase points, and the integral decreases faster than any inverse power of $\tau$. If $\alpha<|t|$, then there are two solutions, and they satisfy the conditions $\pi \leq \theta-\eta \leq 2 \pi$ and

$$
\sin ^{2}(\theta-\eta)=\frac{(\alpha|t|)^{2}}{\left(1-\alpha^{2}\right)|t|^{2}}
$$

Explicitly, if $\gamma$ lies between 0 and $\pi / 2$ and satisfies

$$
\sin ^{2}(\gamma)=\frac{(\alpha|t|)^{2}}{\left(1-\alpha^{2}\right)|t|^{2}}
$$

then the two solutions to Eq. (52) are $\theta_{1}=\eta+\gamma+\pi$ and $\theta_{2}=\eta+2 \pi-\gamma$. We find that

$$
\begin{aligned}
& \omega_{+}^{\prime \prime}\left(\theta_{1}\right)=-\frac{1}{|r|}\left(|t|^{2}-\alpha^{2}\right)^{1 / 2}\left(1-\alpha^{2}\right) \\
& \omega_{+}^{\prime \prime}\left(\theta_{2}\right)=\frac{1}{|r|}\left(|t|^{2}-\alpha^{2}\right)^{1 / 2}\left(1-\alpha^{2}\right),
\end{aligned}
$$

and $\omega_{+}\left(\theta_{1}\right)=\pi-\nu$ and $\omega_{+}\left(\theta_{2}\right)=\nu$, where $0 \leq \nu \leq \pi / 2$ and

$$
\nu=\tan ^{-1}\left(\frac{|r|}{\left(|t|^{2}-\alpha^{2}\right)^{1 / 2}}\right) .
$$

Finally for the integral we find that

$$
\begin{array}{r}
\int_{0}^{2 \pi} d \theta e^{i \tau\left[\alpha \theta+\omega_{+}(\theta)\right]} \frac{|r|^{2}}{4 \pi C(\theta)[C(\theta)+S(\theta)]} \\
\sim \frac{1}{2(1-\alpha)}\left[\frac{|r|\left(1-\alpha^{2}\right)}{2 \pi \tau\left(|t|^{2}-\alpha^{2}\right)^{1 / 2}}\right]^{1 / 2} e^{i \alpha \tau \eta}\left[(-1)^{\tau} e^{i \tau[\alpha(\pi+\gamma)-\nu]-i \pi / 4}\right. \\
\left.+e^{i \tau[\alpha(2 \pi-\gamma)+\nu]+i \pi / 4}\right] .
\end{array}
$$

Finding the asymptotic form of the other integrals in a similar fashion, we have for $j=\alpha \tau$ and $\alpha<|t|$

$$
\begin{aligned}
p(j, j+1 ; \tau) \sim & \frac{|r|}{\pi \tau\left(|t|^{2}-\alpha^{2}\right)^{1 / 2}(1-\alpha)}\left[1+\alpha(-1)^{\tau} \cos (\pi \alpha \tau)\right] \\
& \left\{1+(-1)^{\tau} \sin [2 \tau(\alpha \gamma-\nu)-\pi \alpha \tau]\right\} .
\end{aligned}
$$




\section{References}

[1] A. Nayak and A. Vishwanath, quant-ph/0010117 (2000).

[2] D. Aharonov, A. Ambainis, J. Kempe, and U. Vazirani, quant-ph/0012090 (2000).

[3] T. D. Mackay, S. D. Bartlett, L. T. Stephenson, and B. C. Sanders, Phys. A 35, 2745 (2002).

[4] T. Yamasaki, H. Kobayashi, and H. Imai, quant-ph/0205045 (2002).

[5] E. Bach, S. Coppersmith, M. Paz Goldschen, R. Joynt, and J. Watrous, quant-ph/0207003

[6] C. Moore and A. Russell, quant-ph/0104137 (2001).

[7] J. Kempe, quant-ph/0205083 (2002).

[8] N. Shenvi, J. Kempe, and K. B. Whaley, quant-ph/0210064

[9] T. A. Brun, H. A. Carteret, and A. Ambainis, quant-ph/0208195

[10] V. Kendon and B. Treganna, quant-ph/0209005

[11] A. Childs, E. Farhi, and S. Gutmann, Quantum Information Processing 1, 35 (2002).

[12] D. Meyer, J. Stat. Phys. 85, 551 (1996).

[13] P. Törmä and I. Jex, J. Opt. B 1, 8 (1999).

[14] A. Zeilinger, H. J. Bernstein, D. M. Greenberger, M. A. Horne, and M. Zukowski, Controlling Entanglement in Quantum Optics, in Quantum Control and Measurement, H. Ezawa and Y. Murayama, eds. (North Holland, Amsterdam, 1993).

[15] A. Childs, R. Cleve, E. Deotto, E. Farhi, S. Gutman, and D. Spielman, quant-ph/0209131

[16] J. Watrous, Proc. 33rd Symposium on the Theory of Computing (ACM Press, New York, 2001), p. 60.

[17] C. Bender and M. Orszag, Advanced Mathematical Methods for Scientists and Engineers (McGraw-Hill New York 1978). 